\title{
Anomalous Left Main Coronary Artery Compression Between the Aorta and Pulmonary Artery Presenting as Acute Coronary Syndrome
}

\author{
Diego Celli ${ }^{1}$, Marina Byer ${ }^{2}$, and Cesar Mendoza ${ }^{3}$ \\ ${ }^{1}$ University of Miami Health System \\ ${ }^{2}$ University of Miami Miller School of Medicine \\ ${ }^{3}$ Jackson Memorial Hospital
}

November 10, 2020

\begin{abstract}
We present a case of a 40-year-old male admitted with a non-ST segment elevation myocardial infarction and found to have an anomalous origination of the left main coronary artery from the opposite sinus, a congenital disorder associated with a significant risk of sudden cardiac death.
\end{abstract}

\section{Anomalous Left Main Coronary Artery Compression Between the Aorta and Pulmonary Artery Presenting as Acute Coronary Syndrome}

Author: Diego Celli, MD ${ }^{1}$, Marina Byer, $\mathrm{MD}^{1}$ and Cesar E. Mendoza, MD2.

Affiliations: ${ }^{1}$ Internal Medicine, University of Miami Miller School of Medicine/Jackson Memorial Hospital, Miami, Florida; ${ }^{2}$ Division of Cardiovascular Disease, Jackson Memorial Hospital, Miami, Florida.

Affiliation addresses: ${ }^{1} 1611$ NW 12th Ave 33136, Miami, Florida, United States; ${ }^{2} 1801$ NW $9{ }^{\text {th }}$ Ave, Suite \#209 33136 Miami, Florida, United States.

Corresponding author: Diego Celli, MD; diego.celli@jhsmiami.org; 480 NE 31st St, apt 3102, Miami Fl 33137. Twitter: @celli_md.

Disclosures: Authors have no relationships with the industry. This work is not under consideration in any other journal.

Funding: No grants, contracts, and other forms of financial support were used to perform this manuscript.

Word count: 514

\section{Abstract:}

We present a case of a 40-year-old male admitted with a non-ST segment elevation myocardial infarction and found to have an anomalous origination of the left main coronary artery from the opposite sinus, a congenital disorder associated with a significant risk of sudden cardiac death.

\section{Case:}

A 40-year-old male with no significant history presented to our institution with acute chest pain. His electrocardiogram (ECG) demonstrated incomplete right bundle branch block and no acute ischemic changes. His initial troponin-I was elevated to $1.02 \mathrm{ng} / \mathrm{mL}$. He was diagnosed with a non-ST segment elevation 
myocardial infarction and admitted to the hospital. Transthoracic echocardiogram showed a preserved biventricular function and no regional wall motion abnormalities. Other echocardiographic parameters were normal as well. Patient was placed on current guideline based medical therapy for acute coronary syndrome with subsequent symptomatic relief.

Coronary angiography revealed an anomalous left main coronary artery (LMCA) arising from the right coronary cusp (Figure 1A). Given the high suspicion for a malignant LMCA course, cardiac computed tomography was performed demonstrating an extramural and inter-arterial LMCA trajectory between the ascending aorta and the pulmonary trunk before reaching the anterior sulcus (Figure 1B). Furthermore the LMCA appeared to experience notable compression of $37 \%$ during systole, with caliber reduction from $2.41 \mathrm{~mm}$ in diastole (Figure 1C) to $1.53 \mathrm{~mm}$ in systole (Figure 1D). Cardiothoracic surgery recommended coronary bypass graft to left anterior descending artery to prevent further complications. Patient decided to defer surgery for later. He was recommended to avoid strenuous physical activities and discharged on treatment with aspirin $81 \mathrm{mg}$ daily, metoprolol succinate $50 \mathrm{mg}$ daily and sublingual nitroglycerin for occasional acute chest pain. He agreed to follow up closely in the outpatient cardiology clinic.

Anomalous coronary artery from the opposite sinus (ACAOS) congenital disorders entails a diverse group with varied clinical characteristics and outcomes. Left-ACAOS has been described as the most dangerous occurrence. Indeed, in a continuous young population series from the American Armed Forces Institute of Pathology, 33\% of all cardiac-related deaths were related to ACAOS and specifically to the LMCA with no other anomalies resulting in adverse outcomes ${ }^{1}$. Diverse retrospective studies have determined leftACAOSs low incidence, including the largest one to date reporting $0.017 \%$ of all cardiac angiographies reviewed ${ }^{2}$. Most ACAOS patients either die suddenly associated with strenuous exercise or have an indolent course with atypical chest pain presentation where diagnosis is made later in life ${ }^{3}$. Treatment options for symptomatic ACAOS patients involve medical treatment, coronary angioplasty with stent deployment and surgical correction ${ }^{3}$. In cases of left-ACAOS with malignant course, surgery is usually recommended; nevertheless, multidisciplinary heart team discussion and share decision making with the patient and family members is crucial.

\section{References:}

1. Eckart RE, Scoville SL, Campbell CL, et al. Sudden death in young adults: a 25-year review of autopsies in military recruits. Ann Intern Med . 2004;141(11):829-834. doi:10.7326/0003-4819-141-11-200412070-00005

2. Yamanaka O, Hobbs RE. Coronary artery anomalies in 126,595 patients undergoing coronary arteriography. Cathet Cardiovasc Diagn . 1990;21(1):28-40. doi:10.1002/ccd.1810210110

3. Angelini P. Coronary artery anomalies: an entity in search of an identity. Circulation . 2007;115(10):12961305. doi:10.1161/CIRCULATIONAHA.106.618082

\section{Figure 1:}

Fluoroscopic image depicting contrast administration in right coronary cusp suggesting anomalous origination of left coronary artery B) Cardiac computed tomography angiography depicting extramural and inter-arterial left coronary artery trajectory between the ascending aorta and the pulmonary trunk. Left main coronary artery compression C) $2.41 \mathrm{~mm}$ in diastole D) $1.53 \mathrm{~mm}$ in systole. 


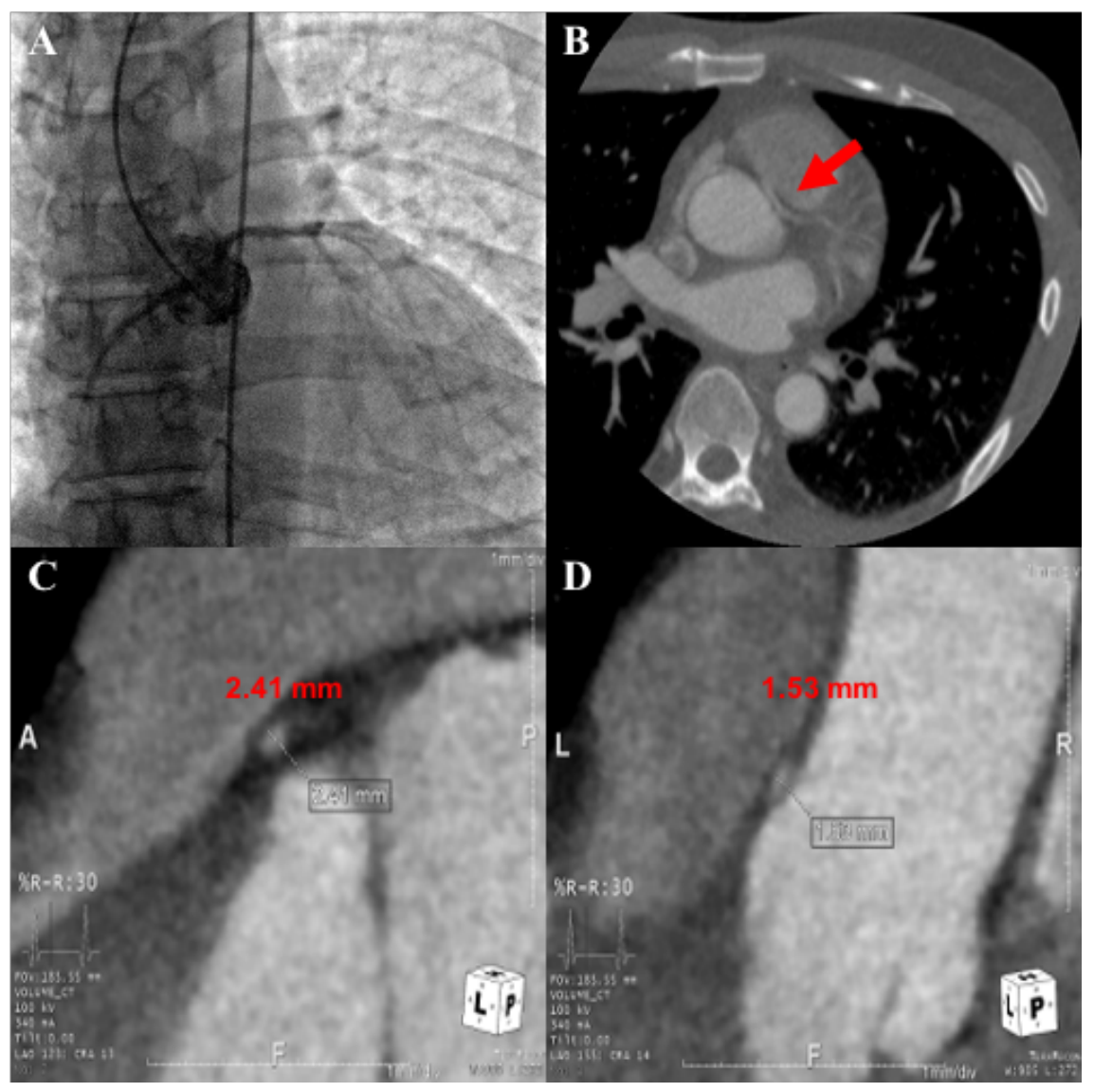

